\title{
Novel Local Bone Void Filling Antibiotic Carriers for the Treatment of Bone Infection
}

\author{
Raquib Hasan and Amanda Brooks* \\ Department of Pharmaceutical Sciences, USA \\ *Corresponding author: Amanda Brooks, Department of Pharmaceutical Sciences, Fargo, ND, 58102, USA
}

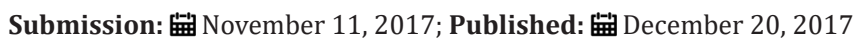

\begin{abstract}
To combat acute and chronic osteomyelitis, particularly implant-associated osteomyelitis, local antibiotic delivery from a biodegradable carrier is favorable over systemic antibiotic treatment. Not only can this help to achieve high local concentration over a desired therapeutic window, but it can also fill the void space left after surgical debridement.This mini review will briefly consider the advantages and disadvantages of synthetic polymer-based materials, ceramics, and calcium-based materials such as calcium phosphate (CP), calcium sulphate (CS), and bioglass, which can be used as a local antibiotic carrier as well as a bone void filler.
\end{abstract}

\section{Introduction}

Although the rates of osteomyelitis or bone infection are relatively low in the controlled surgical environment such as during initial total joint replacement surgeries (TJR), orthopedic infection following trauma, particularly during open fracture [1,2] or revision TJR surgeries [3] are exponentially higher and impose both a personal and societal economic burden [4]. Furthermore, chronic, recurring osteomyelitis in which the causative organism evades both the clinical treatment and the host immune system by residing in the cells, is almost insurmountably more difficult to treat when compared to acute osteomyelitis. Current strategies to treat these infections involve surgical debridement followed by 4 to 6 weeks of intravenous antibiotic therapy with a transition to oral antibiotic therapy for up to 8 weeks $[5,6]$. Unfortunately, due to limited bone vascularization, high systemic antibiotic doses are needed to achieve local concentrations at the infection site above the Minimum inhibitory concentration (MIC), leading to systemic toxicity. Alternatively, to achieve high and effective local antibiotic concentration over longer period of time (4 to 6 weeks) and to fill up the void space left after surgical debridement or orthopedic trauma, biomaterial based local antibiotic delivery is an attractive option [7]. Currently, antibiotic-leaching polymethylmethacrylate (PMMA) bone cement (ALBC) and beads, which may contain either single (e.g., tobramycin or gentamicin) or multiple antibiotics, are a widely used, non-biodegradable option for revision surgeries [8]. Although the use of ALBC has had a modest effect on decreasing post-operative infection, its use is currently only approved in the United States for revision procedures. Regrettably ALBC suffers from poor release kinetics and leach antibiotic at sub-inhibitory levels over an extended period of time [9], leaving the cement to act as a nidus of infection and requiring an additional surgery to remove the cement and/or, beads. Reports indicate that up to $80 \%$ of gentamicin remained in ALBC even after several years of slow leaching [9]. More importantly, inadequate pharmacokinetics may exacerbate the evolution of antibiotic resistant bacteria and put this vulnerable population at risk. One study found that in cases where gentamicin containing ALBC was used in TJR, development of gentamicin resistant bacteria was observed in $88 \%$ of the cases [8]. Moreover, adding antibiotic negatively affects the mechanical property of bone cements, variably [8]. These disadvantages have led to a search for biodegradable bone void filling materials that provide both osteoconduction and osseointegration as well as the ability to act as an antibiotic delivery vehicle. These materials can be primarily divided into calcium-based materials such as hydroxyapatite, calcium phosphate or calcium sulphate-based materials and bioactive glass or bioglass etc. In this short review, we focused mainly on these biodegradable materials as bone void fillers for local antibiotic delivery and bone regeneration.

\section{Calcium Sulphate based material:}

Calcium sulphate (CS) hemihydrate has been used as a resorbable bone substitute. Typically, it has been used as a paste, which when mixed with antibiotic, settles in-situ filling up the void space [10]. Alternatively, calcium sulfate can be used to form antibiotic loaded beads ex-vivo, which can subsequently be implanted inside an orthopedic void space [11]. In a study, where vancomycin loaded calcium sulphate hemihydrate/hydroxyapatite/ collagen was used in a rabbit femoral infection model, the surgical site not only showed no signs of residual infection after 12 weeks but also showed concurrent trabecular bone formation [10]. Although 
this is a promising new tool in the fight against osteomyelitis, the pharmacokinetics of drug release were not assessed and the potential for antibiotic resistant bacteria to arise should be evaluated. Furthermore, gentamycin and vancomycin loaded calcium sulphate beads were clinically evaluated in a metatarsal amputation due to infection [11]. In the study, the infection was eradicated and the patient remained infection free after 11 months. Although this study seems like a stunning example of the efficacy of calcium sulfate beads, it must be noted that the patient also received 4 to 8 weeks of oral antibiotics. In another small clinical study, 4 patients with either revision knee or hip replacement surgery associated with infection were treated with vancomycin or daptomycin loaded CS beads along with antibiotic loaded bone cement (in case of hip surgery)which resulted in complete infection resolution in three patients after 19 months of follow up [12]. Again, all patients in the study received 2 to 4 weeks of intravenous and oral antibiotic therapy in addition to the local implant, which limits the power of the study. Furthermore, these clinical evaluations showed CS absorption after 4 weeks in these cases. In some of these evaluations, antibiotic was loaded in CS beads by soaking the beads in the antibiotic solution. Unfortunately, this passive diffusion often leads to inadequate pharmacokinetics, as was observed when a calcium sulphate and hydroxyapatite composite bead was used as antibiotic carrier for a gentamicin and vancomycin antibiotic solution, which released almost all the drug within only 10 days. Notably, the composite showed faster release when compared to pure CS [13]. Regardless of the antibiotic loading strategy, using CS as a bone substitute incurs several significant drawbacks, including cytotoxicity and an increased acidic microenvironment due to degradation of the CS. Importantly, degradation can also lead to both acute and unresolved chronic inflammation . Furthermore, the exothermic reaction that converts solid CS into a paste can also be locally cytotoxic $[10,13,14]$.

\section{Calcium Phosphate based material}

Compared to CS, calcium phosphate (CP) based materials show longer resorption time. Depending on the composition, CP-based materials have different resorption times. Tricalcium phosphate (TCP) is resorbed in 6 to 18 months, whereas hydroxyapatite (HA) and monocalcium phosphate have degradation times of 6 months to 10 years [14]. Similar to CS, CP can also be used as paste, which gradually solidifies into a solid cement in vivo; however, unlike $\mathrm{CS}$, the process is isothermal, protecting the tissues surrounding the implant. Nevertheless, limited porosity after CP cement solidification leads to limited bone ingrowth [15] and inadequate pharmacokinetics. In a study using gentamicin and vancomycin soaked tricalcium phosphate ceramic, Cerasorb® (low porosity, $35 \%$ ) and Cerasorb® M (high porosity, 65\%), it was observed that both of the antibiotics released from the pellets reached therapeutic high local concentration within 4 to 5 days, but quickly fell below minimum inhibitory concentration (MIC) [16]. In an alternative preclinical study of a CP-based antibiotic eluting bone void filler (ABVF), a porous CP based substrate was embedded in a polymer matrix loaded with tobramycin and showed tobramycin release at therapeutic concentrations up to 8 weeks in vitro [17] and infection control with promising bone formation after 12 weeks in a rabbit radial infection model. Subsequently, the CP-based coralline Proosteon $^{\text {TM }}$ (Biomet) was formulated into an antibiotic releasing bone void filling (ABVF) putty by embedding Pro-osteon ${ }^{\mathrm{TM}}$ granules into different custom or commercial polymers with vancomycin. Dependent on the polymer matrix vancomycin was able to provide antibacterial activity over 6 weeks in vitro [18]. Putty like ABVF has a distinct advantage over its solid and bead-like cousin formulations in that it provides intimal contact with the host bone. CP based materials are promising to be used as antibiotic carrier and as bone void filler; however, further improvements in the formulations are necessary to achieve high local antibiotic concentration over longer period of time.

\section{Bioactive Glass}

As opposed to traditional calcium based bone graft substitutes, recent years have seen bioactive glass or, bioglass garner much attention as a bone substitute for both its osteoconductive and osteoinductive activity as well as its tailorability [19] and antibacterial properties [20]. In a study of 116 chronic osteomyelitis patients treated with antibacterial bioglass, S53P4, 90\% of the patients were infection free after a median follow-up of 31 months [20]. In another study where vancomycin was loaded onto borate bioglass pellets in vitro drug release above the minimum inhibitory concentration (MIC) was observed for up to 18 days. Subsequently, the vancomycin loaded bioglass pellets were used in a rabbit tibial infection model. After 11 weeks, $81.25 \%$ of the animals showed no signs of infection along with good bone growth and osseointegration [21]. In another study, borate bioglass was mixed with chitosan to form a injectable paste, which was subsequently loaded with vancomycin. In vitro, this paste like composition showed drug release up to 36 days above MIC. In vivo, the composition showed eradication of infection in 14 out of 16 rabbits 2 months post-surgery in a rabbit tibial infection model. Importantly, the antimicrobial activity of the bioglass paste may have been confounded with the use of chitosan, which itself has antimicrobial activity [22]. Nevertheless, the paste also showed both osteoconduction and osseointegration [23]. Ultimately, bioglass has been increasingly shown as a promising bone substitute and antibiotic carrier to treat osteomyelitis.

\section{Conclusion}

Orthopedic surgeries represent a significant portion of surgical procedures. A variety of calcium and ceramic bone graft void fillers have been used to not only promote osseointegration but also as a drug delivery carrier. However, the success of bone graft in osteointegration and antibiotic release can be correlated with the porosity of bone graft substrate [24,25]. Several highly porous CPbased bone void fillers have been shown effective for both in vitro drug release and in vivo infection protection and osseointegration.

\section{References}

1. Fernandes M de C, Peres LR, de Queiroz AC, Lima JQ Turíbio FM, et al. (2015) Open fractures and the incidence of infection in the surgical debridement 6 hours after trauma. Acta Ortop Bras 23(1): 38-42. 
2. Doshi P, Gopalan H, Sprague S, Pradhan C, Kulkarni S, et al. (2017) Incidence of infection following internal fixation of open and closed tibia fractures in India (INFINITI): a multi-centre observational cohort study. BMC Musculoskelet Disord 18(1): 156

3. Mortazavi SMJ, Schwartzenberger J, Austin MS, Purtill JJ, Parvizi J, et al. (2010) Revision Total Knee Arthroplasty Infection: Incidence and Predictors. Clin Orthop 468(8): 2052-2059.

4. Kurtz SM, Lau E, Watson H, Schmier JK, Parvizi J (2012) Economic Burden of Periprosthetic Joint Infection in the United States. J Arthroplasty 27(8 Suppl): 61-65.e1

5. Kalore NV, Gioe TJ, Singh JA (2011) Diagnosis and Management of Infected Total Knee Arthroplasty. Open Orthop J 5: 86-91.

6. Haidar R, Boghossian AD, Atiyeh B (2010) Duration of post-surgica antibiotics in chronic osteomyelitis: empiric or evidence-based? Int J Infect Dis 14(9): e752-e758.

7. Gogia JS, Meehan JP, Di Cesare PE, Jamali AA (2009) Local Antibiotic Therapy in Osteomyelitis. Semin Plast Surg 23(2): 100-107.

8. Bistolfi A, Massazza G, Verné E, Massè A, Deledda D, et al. (2011) Antibiotic-Loaded Cement in Orthopedic Surgery: A Review. Int Sch Res Not p. e290851.

9. Hendriks JGE, van Horn JR, van der Mei HC, Busscher HJ (2004) Backgrounds of antibiotic-loaded bone cement and prosthesis-related infection. Biomaterials 25(3): 545-556.

10. Lian X, Mao K, Liu X, Wang X, Cui F, et al. (2015) In Vivo Osteogenesis of Vancomycin Loaded Nanohydroxyapatite/Collagen/Calcium Sulfate Composite for Treating Infectious Bone Defect Induced by Chronic Osteomyelitis. Journal of Nanomaterials Article ID: 261492

11. Morley R, Lopez F, Webb F (2016) Calcium sulphate as a drug delivery system in a deep diabetic foot infection. Foot (Edinb) 27: 36-40.

12. The use of antibiotic impregnated absorbable calcium sulphate beads in management of infected joint replacement prostheses.

13. Rauschmann MA, Wichelhaus TA, Stirnal V, Dingeldein E, Zichner L, et al. (2005) Nanocrystalline hydroxyapatite and calcium sulphate as biodegradable composite carrier material for local delivery of antibiotics in bone infections. Biomaterials 26(15): 2677-2684.

14. Ferguson J, Diefenbeck M, McNally M (2017) Ceramic Biocomposites as Biodegradable Antibiotic Carriers in the Treatment of Bone Infections. J
Bone Jt Infect 2(1): 38-51.

15. M Bohner (2000) Calcium orthophosphates in medicine: from ceramics to calcium phosphate cements. Injury 31( Suppl 4): 37-47.

16. Maier GS et al. (2013) In Vitro Elution Characteristics of Gentamicin and Vancomycin from Synthetic Bone Graft Substitutes. Open Orthop J 7: 624-629.

17. Jones Z, Brooks AE, Ferrell Z, Grainger DW, Sinclair KD (2016) A resorbable antibiotic eluting bone void filler for periprosthetic joint infection prevention. J Biomed Mater Res B Appl Biomater 104(8): 1632 1642

18. Curley J, Hasan MR, Larson J, Brooks BD, Liu Q, et al. (2016) An Osteoconductive Antibiotic Bone Eluting Putty with a Custom Polymer Matrix. Polymers 8(7): 247.

19. Rahaman MN, Day DE, Bal BS, Fu Q, Jung SB, et al. (2011) Bioactive glass in tissue engineering. Acta Biomater 7(6): 2355-2373.

20. Lindfors N, Geurts J, Drago L, Arts JJ, Juutilainen V, et al. (2017) Antibacterial Bioactive Glass, S53P4, for Chronic Bone Infections-A Multinational Study. A Modern Approach to Biofilm-Related Orthopaedic Implant Infections pp. 81-92.

21.Xie Z, Liu X, Jia W, Zhang C, Huang W, et al. (2009) Treatment of osteomyelitis and repair of bone defect by degradable bioactive borate glass releasing vancomycin. J Controlled Release 139(2): 118-126.

22. Muñoz-Bonilla A, Cerrada ML, Fernández-García M (2013) CHAPTER 2. Antimicrobial Activity of Chitosan in Food, Agriculture and Biomedicine. In: Muñoz-Bonilla A, Cerrada M, Fernández-García M, (Eds.), RSC Polymer Chemistry Series. Royal Society of Chemistry, Cambridge, USA, pp. 22-53.

23. Ding H, Zhao CJ, Cui X, Gu YF, Jia WT, et al. (2014) A Novel Injectable Borate Bioactive Glass Cement as an Antibiotic Delivery Vehicle for Treating Osteomyelitis. Plos One 9(1): e85472.

24. Baino F, Fiorilli S, Vitale-Brovarone C (2016) Bioactive glass-based materials with hierarchical porosity for medical applications: Review of recent advances. Acta Biomater 42: 18-32.

25. Paulo MJE, dos Santos MA, Cimatti B, Gava NF, Riberto M, et al. (2017) Osteointegration of porous absorbable bone substitutes: A systematic review of the literature. Clinics 72(7): 449-453. 